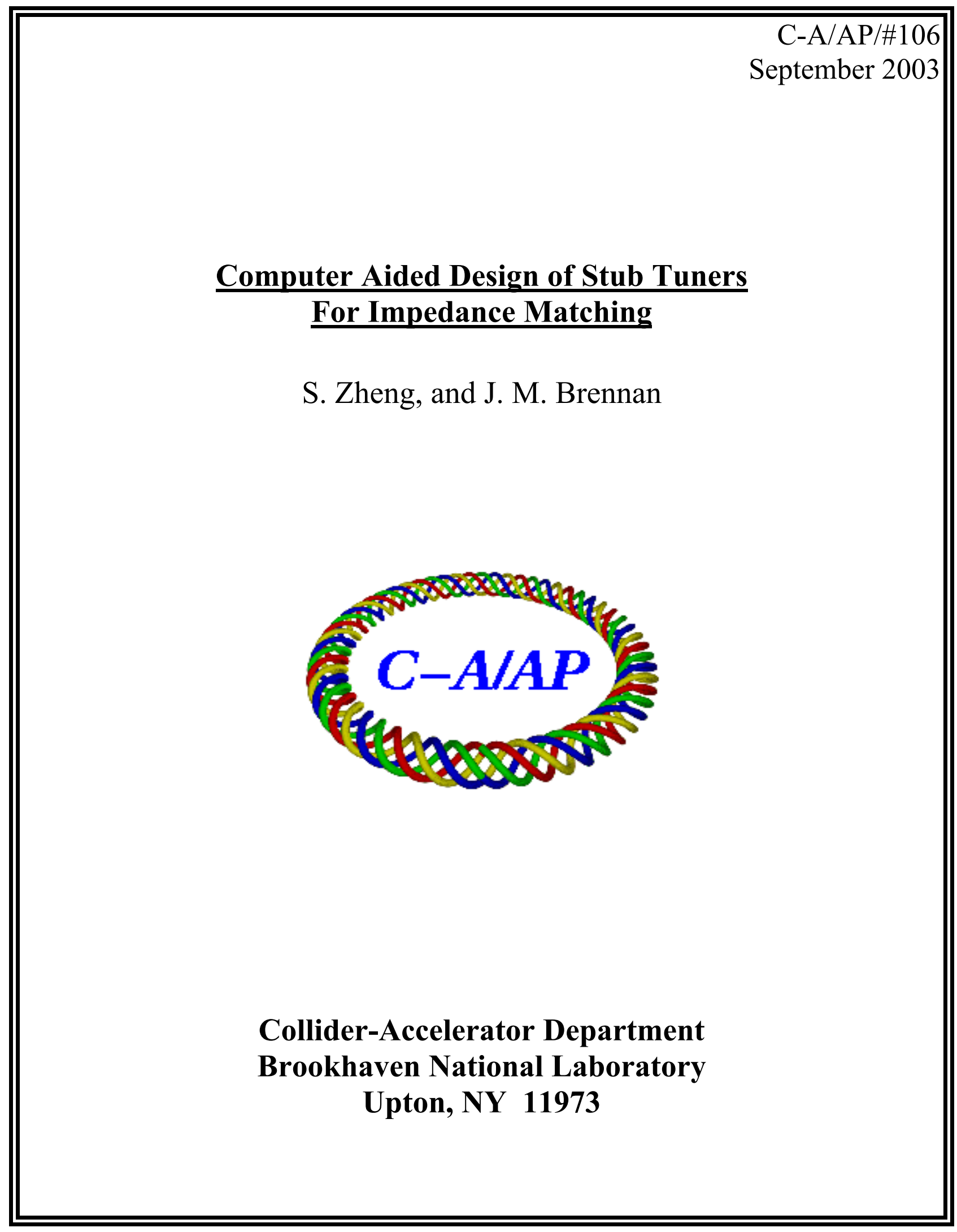




\title{
Computer Aided Design of Stub Tuners For Impedance Matching
}

\author{
S. Zheng and J. M. Brennan
}

\section{I}

\section{Introduction}

In RF and microwave circuit design, a common situation is to match a load with arbitrary impedance $Z_{L}$ to an input circuit with a different impedance $Z_{S}$. Several techniques for this impedance matching are available. Among them, quarter wavelength transmission line with characteristic impedance given by $Z_{0}=\sqrt{Z_{L} Z_{S}}$ is well known. This method, however, is difficult to implement in high power applications since $Z_{0}$ obtained from the impedance equation is usually a non-standard value.

Another matching technique, namely stub tuner [1], can be used to solve the problem. This method uses two pieces of transmission lines, thus the impedance of the matching transmission lines can be arbitrarily chosen and commercially available cables are usually used to form the matching network. This stub tuner was proposed to replace the old quarter wavelength matching cable in the RHIC $28 \mathrm{MHz}$ accelerating PA input circuits. The design of the stub tuner using MATLAB is given in this note.

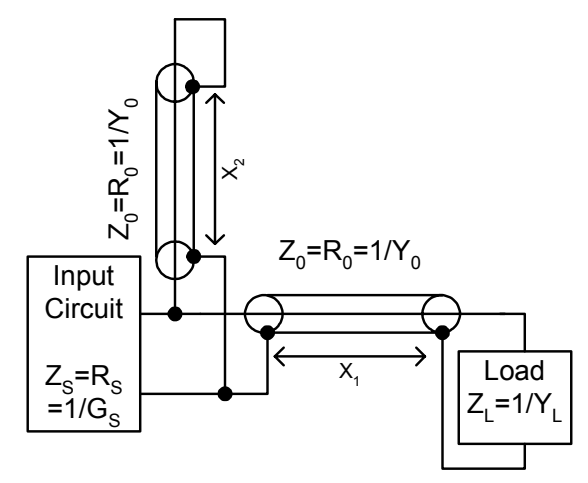

Fig. 1 A stub matching network.

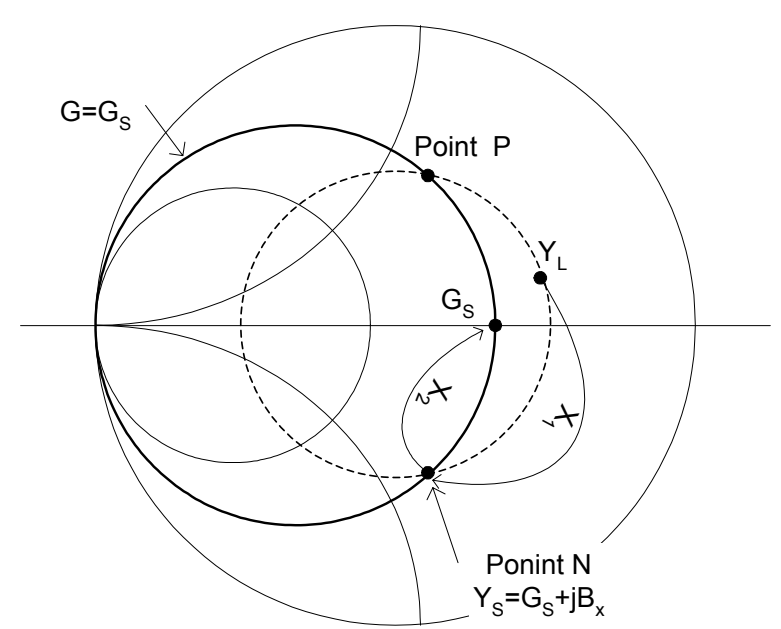

Fig. 2 Smith chart explanation of the stub tuner.

\section{II}

\section{Stub length calculation}

A stub tuner is shown in Fig. 1, where $X_{1}$ and $X_{2}$ are the normalized length of the matching cables, and the shunt-connected transmission line is shorted at one end. The input circuit is assumed to be a lossless transmission line with a characteristic impedance $R_{S}$ or a circuit with a pure resistive output impedance $R_{S}$.

The basic idea of the stub tuner is to transform the load admittance $Y_{L}$ (normalized to $Y_{0}$ ) to a point $Y_{x}=G_{S}+j B_{x}$ on the admittance circle $G=G_{S}$ of the smith chart using cable 
$\mathrm{X}_{1}$, and then compensate the susceptance part $B_{x}$ by the shorted stub $\mathrm{X}_{2}$ to obtain a match to the input admittance $G_{S}$, as shown in Fig. 2.

Without consideration of the shorted stub, the admittance looking from the source end of $\mathrm{X}_{1}$ is (Assume lossless matching cable):

$$
Y_{x}=\frac{Y_{L}+j \tan \left(2 \pi X_{1}\right)}{1+j Y_{L} \tan \left(2 \pi X_{1}\right)}
$$

Let $Y_{x}=G_{S}+j B_{x}$ and $Y_{L}=G_{L}+j B_{L}$. Solving $\tan \left(2 \pi X_{1}\right)$ from the above equation one gets:

$$
\begin{aligned}
\tan \left(2 \pi X_{1}\right)= & \frac{\left(G_{S}-G_{L}\right)\left(B_{L} G_{S}+G_{L} B_{x}\right)-\left(B_{x}-B_{L}\right)\left(G_{L} G_{S}-B_{L} B_{x}-1\right)}{\left(G_{L} G_{S}-B_{L} B_{x}-1\right)^{2}+\left(B_{L} G_{S}+G_{L} B_{x}\right)^{2}} \\
& +j \frac{\left(G_{S}-G_{L}\right)\left(G_{L} G_{S}-B_{L} B_{x}-1\right)+\left(B_{x}-B_{L}\right)\left(B_{L} G_{S}+G_{L} B_{x}\right)}{\left(G_{L} G_{S}-B_{L} B_{x}-1\right)^{2}+\left(B_{L} G_{S}+G_{L} B_{x}\right)^{2}}
\end{aligned}
$$

Since $\tan \left(2 \pi X_{l}\right)$ can not be complex, the imaginary part of the above equation must be zero, and thus $B_{x}$ is solved:

$$
B_{x}= \pm \sqrt{\left(\frac{B_{L}^{2}}{G_{L}}+G_{L}+\frac{1}{G_{L}}-G_{S}\right) G_{S}-1} .
$$

A match can be obtained as long as $B_{x}$ 's are real. Here $B_{x}$ has two values. The positive one is indicated by point $\mathrm{P}$ in Fig. 2. It is an inductive point and needs a capacitive shorted stub (longer than a quarter wave length) to compensate it to $G_{S}$. The negative point (point $\mathrm{N}$ in Fig.2) is a capacitive point and needs an inductive stub (shorter than quarter wavelength) for compensation. However, if the initial point $Y_{L}$ is inside the $G=G_{S}$ circle, the total length of the two pieces of cables with a capacitive stub may be shorter than that with an inductive stub.

Normalized length $X_{1}$ is obtained from the real part of (2):

$$
X_{1}=\frac{1}{2 \pi} \tan ^{-1}\left(\frac{\left(G_{S}-G_{L}\right)\left(B_{L} G_{S}+G_{L} B_{x}\right)-\left(B_{x}-B_{L}\right)\left(G_{L} G_{S}-B_{L} B_{x}-1\right)}{\left(G_{L} G_{S}-B_{L} B_{x}-1\right)^{2}+\left(B_{L} G_{S}+G_{L} B_{x}\right)^{2}}\right) .
$$

The shunt-connected matching cable has an admittance of

$$
Y_{y}=\frac{1}{j \tan \left(2 \pi X_{2}\right)}
$$

Since it is used to compensate $B_{x}$ obtained above, let it equal to $-j B_{x}$. Thus $X_{2}$ is obtained: 


$$
X_{2}=\frac{1}{2 \pi} \tan ^{-1}\left(\frac{1}{B_{x}}\right) .
$$

For convenience, the normalized cable length of $X_{1}$ and $X_{2}$ can be converted to time delay TD:

$$
T D=\frac{X}{f} .
$$

Where $\mathrm{X}$ is the normalized length and $f$ is the frequency.

\section{MATLAB implementation and test result}

MATLAB code has been written to perform the above calculations. It takes the load impedance $Z_{L}$, the impedance of the input circuit $R_{S}$, and the matching cable impedance $R_{0}$ as the inputs, and gives the results for both inductive stub and capacitive stub. The code is given in Appendix I. A plot showing matching cable lengths vs. load resistance for the case of $28 \mathrm{MHz}$ PA is given in Fig.3.

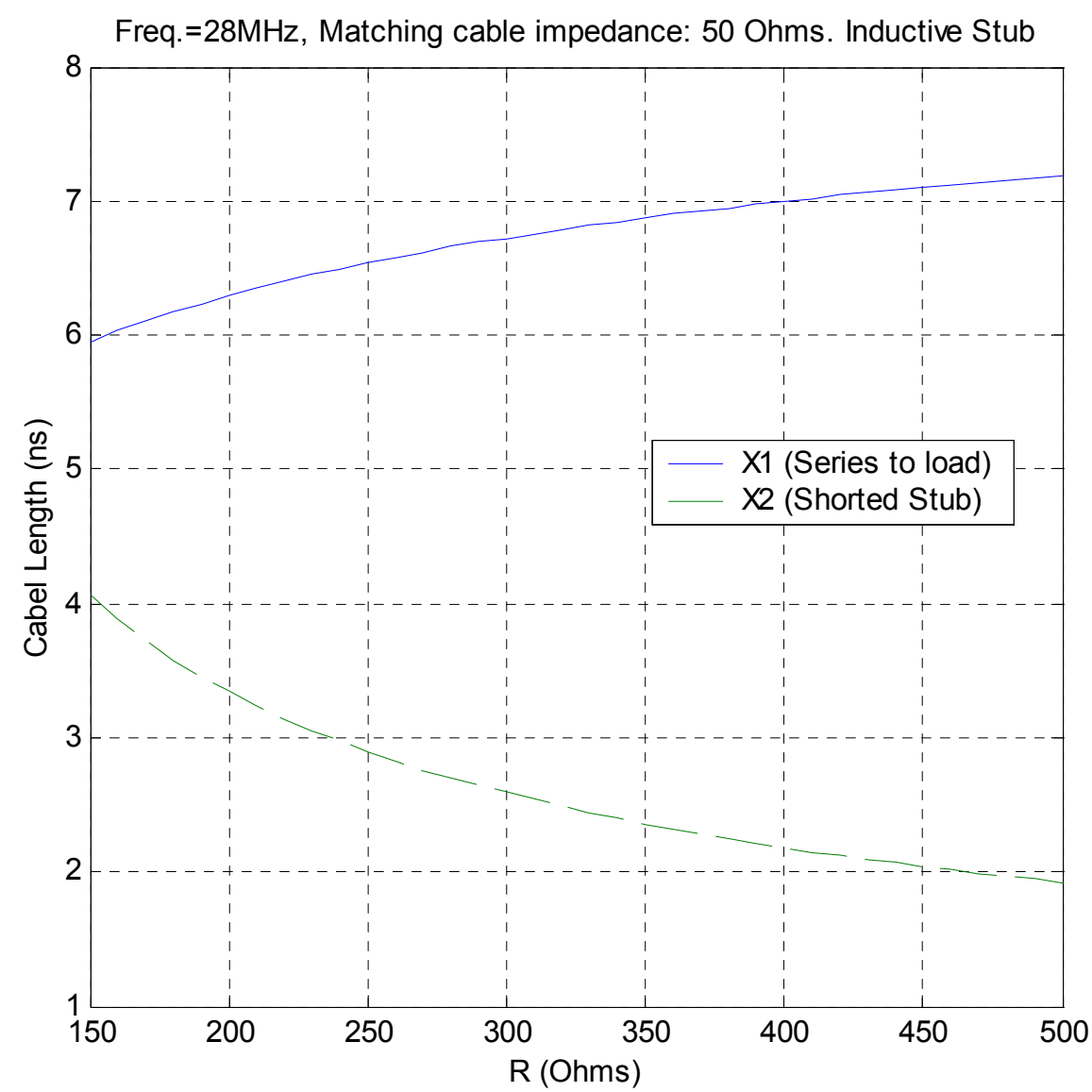

Fig. 3 Designing curves for the $28 \mathrm{MHz}$ PA stub generated by MATLAB. 
A stub tuner is made for a $28 \mathrm{MHz}$ PA. The input resistance of the PA is about 300 $\Omega$. From Fig. 3, the lengths of the stub cables are: $X_{1}=6.7 \mathrm{~ns}, \mathrm{X}_{2}=2.6 \mathrm{~ns}$. The matched input impedance measured by Network Analyzer is shown in Fig. 4.

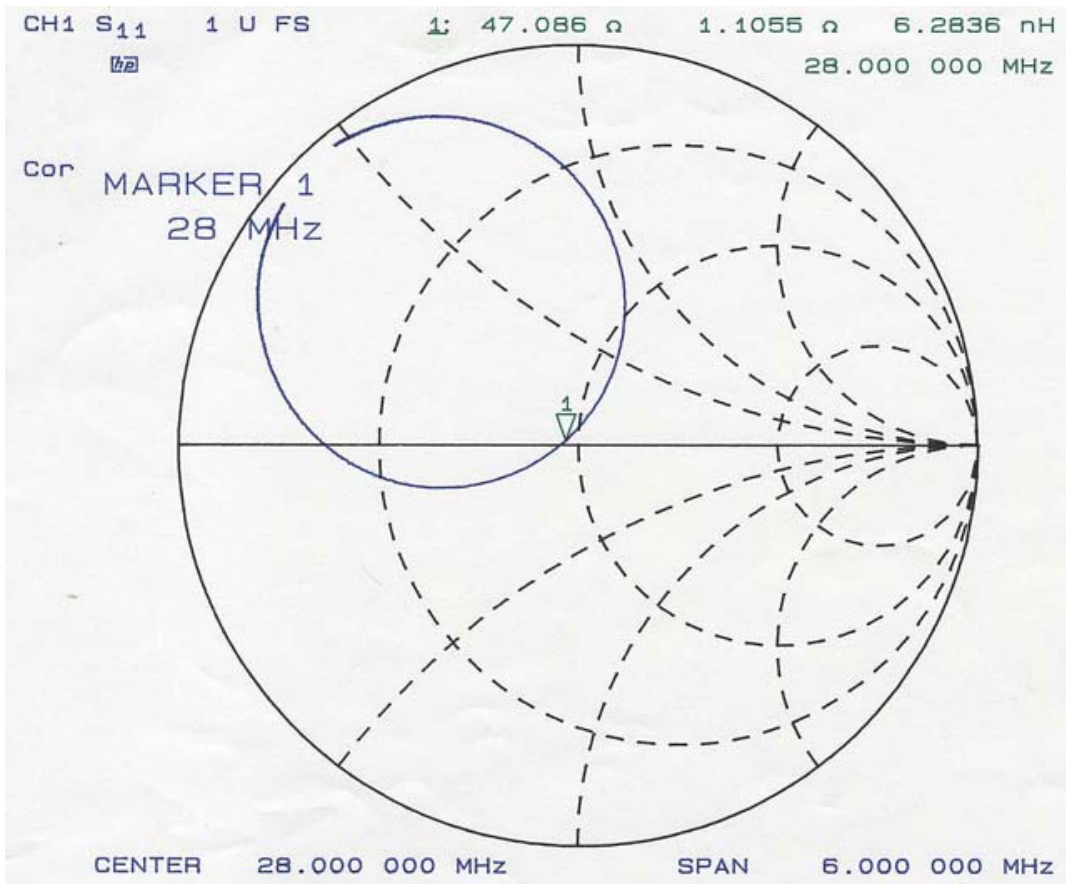

Fig. 4 The $28 \mathrm{MHz}$ PA input impedance looking from the stub tuner.

\section{Summary}

A method of designing stub tuner is present. A MATLAB program is written to perform the calculation. The design has been verified by its application in the $28 \mathrm{MHz}$ PA.

\section{Reference}

[1]John D. Kraus, “Electromagnetics,” Third edition, McGraw-Hill Book Company, 1984.

\section{Appendix MATLAB Program}

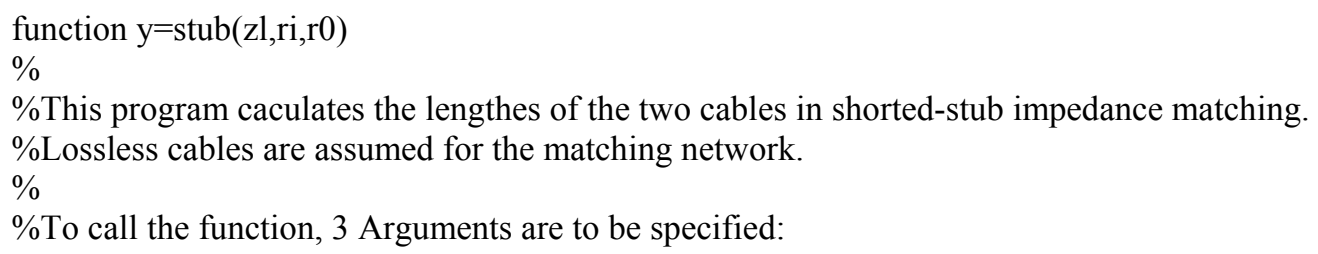


$\%$

$\% \quad z l$ : Load impedance. If it's a complex number, using the form of "R $+\mathrm{j}^{*} \mathrm{X}$;

$\%$ ri : The impedance of the input cable;

$\%$ r0 : The impedance of the matching cables. This parameter can be omitted if it is the

$\% \quad$ same as that of the input cable.

$\%$

$\%$ The output is the cable lengthes (in Wavelength) in a $2 * 2$ matrix. The first row of the

$\%$ matrix shows the cable lengthes with a inductive stub (less than quater-wavelength long).

$\%$ The second row gives the lengthes with a capacitive stub. It has the following form:

$\%$

$\% \quad \mathrm{X} 1$ ind $\mathrm{X} 2$ ind

$\% \quad$ X1_cap X2_cap

$\%$

$\%$ where $\mathrm{X} 1$ is the length of the cable series-connected from the load to the stub,

$\% \mathrm{X} 2$ is the length of the stub. Choice between these two solutions can be made

$\%$ by picking up the one with the shoter total cable length or the one with a shoter stub length.

$\%$

$\%$ Example:

\%Suppose a load of $200 \mathrm{Ohms}$ needs to be matched to an input impedance of $50 \mathrm{Ohms}$ and

$\%$ the matching cables are $50 \mathrm{Ohms}$ :

$\%$

$\%>>\operatorname{stub}(200,50,50)$

$\%$

$\%$ ans $=$

$\%$

$\% \quad 0.1762 \quad 0.0936$

$\% \quad 0.3238 \quad 0.4064$

$\%$ Check the inputs.

if $($ nargin $<2)$

disp('***At least two arguments-load impedance (Ohms), and input cable impedance (Ohms)-are required !');

return;

elseif (nargin $==2)$

$\operatorname{disp}\left({ }^{\prime * * *}\right.$ The impedance of the matching cables are assumed to be equal to that of the input cable

$! ')$

$\mathrm{r} 0=\mathrm{ri}$;

end

if $\mathrm{zl}==\mathrm{ri}$

$\operatorname{disp}\left({ }^{* * *}\right.$ The load impedance already matches the input impedance!');

return;

end

$\%$ Constant caculations and transformation from impedances to admittances.

$\mathrm{Yl}=\mathrm{r} 0 / \mathrm{zl}$

$\mathrm{Gl}=\mathrm{real}(\mathrm{Yl})$;

$\mathrm{Bl}=\operatorname{imag}(\mathrm{Yl})$;

$\mathrm{Gx}=\mathrm{r} 0 / \mathrm{ri}$;

\%Caculation of the suceptance that needs to be compensated by the stub.

$\mathrm{Bx} \_\mathrm{Sq}=\left(\mathrm{Bl}{ }^{\wedge} 2 / \mathrm{Gl}+\mathrm{Gl}+1 / \mathrm{Gl}\right) * \mathrm{Gx}-\mathrm{Gx}^{\wedge} 2-1$;

if $\mathrm{Bx} \_\mathrm{Sq}<0$

disp('***No possible match can be found !');

return; 


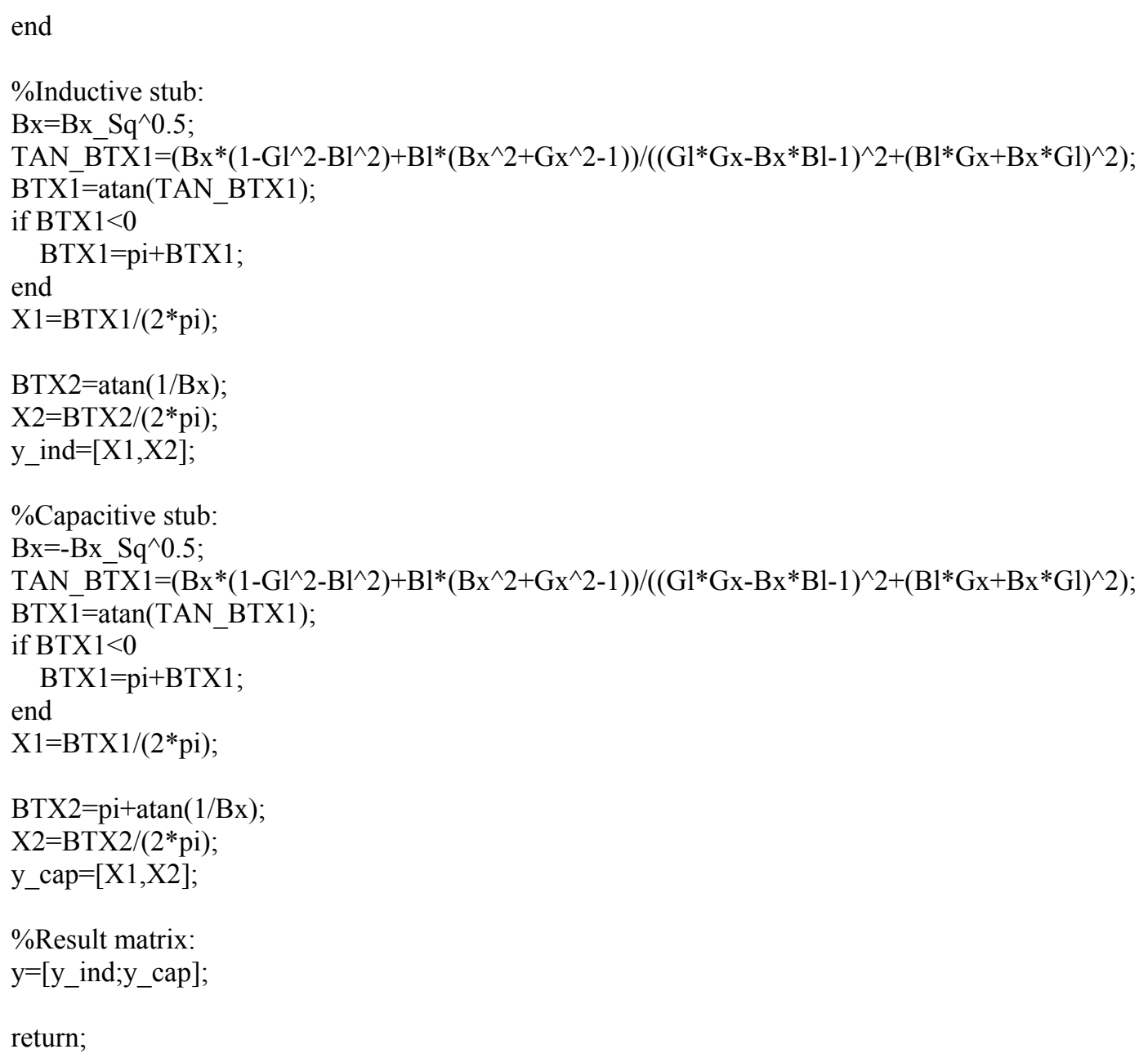

